\section{(B) OPEN ACCESS}

\title{
The cost effectiveness of ivabradine in the treatment of chronic heart failure from the UK National Health Service perspective
}

\author{
A Griffiths, ${ }_{1}^{1}$ N Paracha, ${ }^{1}$ A Davies, ${ }^{1}$ N Branscombe, ${ }^{2}$ M R Cowie, ${ }^{3}$ M Sculpher ${ }^{1,4}$
}

- Additional material is published online only. To view please visit the journal online (http://dx.doi.org/10.1136/ heartjnl-2013-304598).

${ }^{1}$ ICON Health Economics, Oxford, UK

${ }^{2}$ Servier Laboratories Ltd, Suresnes, France

${ }^{3}$ Imperial College London, Royal Brompton Hospital, London, UK

${ }^{4}$ Centre for Health Economics, University of York, York, UK

\section{Correspondence to}

Professor Martin R Cowie, Imperial College London, Dovehouse Street, London SW3 6LY, UK;

m.cowie@imperial.ac.uk

Received 12 July 2013 Revised 16 December 2013 Accepted 9 February 2014 Published Online First 14 March 2014

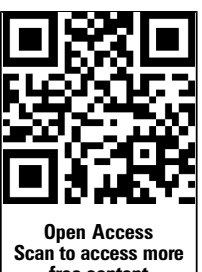
free content

\section{Linked}

http://dx.doi.org/10.1136/ heartjnl-2014-305684

\section{CrossMark}

To cite: Griffiths $A$, Paracha N, Davies A, et al. Heart 2014;100: 1031-1036.

\begin{abstract}
Objective Ivabradine, a specific heart rate lowering therapy, has been shown in a randomised placebocontrolled study, Systolic HF Treatment with the $I_{f}$ Inhibitor Ivabradine Trial $\left(\mathrm{SH}_{\mathrm{f}} \mathrm{T}\right)$, to significantly reduce the composite end point of cardiovascular death and hospitalisation for worsening heart failure (HF) in patients with systolic HF who are in sinus rhythm and with a heart rate $\geq 70 \mathrm{bpm}$, when added to optimised medical therapy (HR: $0.82,95 \% \mathrm{Cl} 0.75$ to 0.90 , $p<0.0001)$. We assessed the cost effectiveness of ivabradine, from a UK National Health Service perspective, based on the results of $\mathrm{SHI}_{\mathrm{f}} \mathrm{T}$.
\end{abstract}

Methods A Markov model estimated the cost effectiveness of ivabradine compared with standard care for two cohorts of patients with HF (heart rate $\geq 75 \mathrm{bpm}$ in line with the EU labelled indication; and heart rate $\geq 70 \mathrm{bpm}$ in line with the $\mathrm{SH}_{\mp} \mathrm{T}$ study population). Modelled outcomes included death, hospitalisation, quality of life and New York Heart Association class. Total costs and quality adjusted life years (QALYS) for ivabradine and standard care were estimated over a lifetime horizon.

Results The incremental cost per additional QALY for ivabradine plus standard care versus standard care has been estimated as $£ 8498$ for heart rate $\geq 75 \mathrm{bpm}$ and f13 764 for heart rate $\geq 70 \mathrm{bpm}$. Ivabradine is expected to have a $95 \%$ chance of being cost-effective in the EU licensed population using the current National Institute for Health and Care Excellence cost effectiveness threshold of $£ 20000$ per QALY. These results were robust in sensitivity analyses.

Conclusions This economic evaluation suggests that the use of ivabradine is likely to be cost-effective in eligible patients with HF from a UK National Health Service perspective.

\section{INTRODUCTION}

Heart failure (HF) is a clinical syndrome characterised by the inability of the heart to pump enough blood to meet the body's demands. Symptoms include dyspnoea and fatigue which may limit exercise tolerance as well as fluid retention which may lead to peripheral oedema and pulmonary congestion. The British Heart Foundation (BHF) estimates that around 750000 people in the UK have HF and there are approximately 25000 new cases each year. Prognosis from HF is poor and the 5 -year survival rate for patients with HF is estimated to be only $58 \% .^{1-3}$
Epidemiological and clinical studies indicate that a higher resting heart rate in sinus rhythm is associated with increased morbidity and mortality in the general population and in patients with cardiovascular $(\mathrm{CV})$ disease. Heart rate reduction is associated with improved outcomes in patients with $\mathrm{HF}^{4}$ and some of the beneficial effects of $\beta$-blockade may be attributed to heart rate reduction. ${ }^{5}$ However, some patients cannot tolerate target dosages of $\beta$-blockers and, when resting heart rates remain elevated despite efforts to optimise $\beta$-blocker dose, there is potential benefit from further heart rate reduction.

Ivabradine is a pure heart rate lowering therapy, which acts by selective and specific inhibition of the cardiac pacemaker current via the $\mathrm{I}_{\mathrm{f}}$ channel. The effect of using ivabradine to slow the heart rate in patients with systolic HF, in addition to standard care medications including $\beta$-blockade, has been examined in a large, randomised, placebocontrolled trial: Systolic HF Treatment with the $\mathrm{I}_{\mathrm{f}}$ Inhibitor Ivabradine Trial $\left(\mathrm{SHI}_{\mathrm{f}} \mathrm{T}\right){ }^{6}$ This trial assessed 6505 patients with symptomatic HF (New York Heart Association (NYHA) classes II to IV), sinus rhythm, and a left ventricular EF $\leq 35 \%$, with a prior hospitalisation for HF within 12 months and a baseline resting heart rate $\geq 70 \mathrm{bpm}$ despite optimised medical therapy. Ivabradine therapy was associated with a significant reduction in the number of primary composite end point events compared with standard care (CV death or hospitalisation for worsening HF; HR: $0.82 ; 95 \%$ CI 0.75 to $0.90, \mathrm{p}<0.0001)$. This result was driven primarily by a reduction in HF hospitalisations (first event worsening HF HR: 0.74; 95\% CI 0.66 to $0.83, \mathrm{p}<0.0001$ ) and HF death (HR: $0.74 ; 95 \%$ CI 0.58 to $0.94, \mathrm{p}=0.014)$. There was also a reduction in overall CV mortality (HF and other CV mortality HR: $0.91 ; 95 \%$ CI 0.80 to 1.03, $\mathrm{p}=0.128$ ), however, this result did not achieve statistical significance.

The ivabradine treatment effect was found to be consistent across most patient subgroups but was modified by baseline heart rate. In patients with a heart rate $\geq 75 \mathrm{bpm}$, a significant treatment effect was also demonstrated on CV mortality (HR: 0.83; 95\% CI 0.71 to $0.97, \mathrm{p}=0.02$ ) as well as all-cause mortality (HR: $0.83 ; 95 \%$ CI 0.72 to 0.96 ). ${ }^{7}$ Standard care treatment patterns in $\mathrm{SHI}_{\mathrm{f}} \mathrm{T}$ appeared at least as good as clinical practice in the UK and elsewhere in Europe, ${ }^{8}$ even though only $26 \%$ of patients achieved the target dose $\beta$-blockade 
considered to represent gold standard $\beta$-blocker therapy in patients with HF. While it is not expected that target dose $\beta$-blockade can be achieved in all patients in clinical practice, due to intolerance to therapy and contraindications to use, it is recognised that $\beta$-blockade lowers resting heart rate and the effect of ivabradine is modified by resting heart rate.

Adoption of new treatment is influenced by an assessment of the relative efficacy and safety of the treatment, and by whether an intervention is likely to represent value for money, assessed using economic evaluation which systematically compares the costs and benefits of a new therapy relative to existing care. A cost effectiveness analysis was developed to compare ivabradine plus standard care versus standard care alone using resource use and clinical outcomes reported in $\mathrm{SHI}_{\mathrm{f}} \mathrm{T}$. The model has been designed to be adapted to a population consistent with the European licensed indication (heart rate $\geq 75 \mathrm{bpm}$ ). The model also presents results for an average population which has been optimised on $\beta$-blocker therapy according to current clinical practice, as well as for a subgroup population treated with target dose $\beta$-blockade given interest in the treatment effect of ivabradine on top of target dose $\beta$-blockade.

\section{METHODS}

\section{Overview}

A Markov model has been used to estimate the costs and clinical outcomes for two cohorts of patients with HF treated with either ivabradine or standard care in line with the EU labelled indication (heart rate $\geq 75 \mathrm{bpm}$ ).

$\mathrm{HF}$ is a chronic, progressive disease requiring lifelong therapy and consequently the cost effectiveness analysis considers a lifetime time horizon, although alternative time horizons, including an analysis which modelled costs and outcomes only for the $\mathrm{SHI}_{\mathrm{f}} \mathrm{T}$ trial follow-up period, have been considered in scenario analyses. An annual discount rate of $3.5 \%$ has been applied to costs and outcomes consistent with the National Institute for Health and Care Excellence (NICE) recommendations. ${ }^{9}$ The cost effectiveness of ivabradine is expressed in terms of the incremental cost per quality adjusted life year (QALY) gained, and the analysis has been taken from a UK National Health Service (NHS) perspective. In order to determine whether ivabradine represented value for money, the incremental cost effectiveness ratio (ICER) was compared with the NICE cost effectiveness threshold range of $£ 20000$ to $£ 30000$ per QALY. $^{9}$ A summary of key model assumptions may be found in table 1 .

\section{Mortality and hospitalisation}

The cost-effectiveness analysis captures the monthly risk of clinical events (CV mortality, hospitalisation) using risk equations developed from $\mathrm{SHI}_{\mathrm{f}} \mathrm{T}$ individual patient data $(\mathrm{n}=6505)$. These equations have been designed to predict outcomes according to the treatment received and patients' baseline characteristics including heart rate. The treatment effect of ivabradine is assumed to be multiplicative to the underlying risk of these events which has been based on the data from standard care patients in $\mathrm{SHI}_{\mathrm{f}} \mathrm{T}$. The change in efficacy of ivabradine associated with baseline heart rate, identified in previous clinical analyses, ${ }^{7}$ is captured in the risk equations using a treatment interaction term (treatment $\times$ baseline heart rate). The risk equations consequently allow costs and outcomes to be predicted for the subgroup of patients with a heart rate $\geq 75 \mathrm{bpm}$, consistent with the European licence indication. This approach was taken in preference to developing risk equations based solely on individual patient data from subjects who met the European licence criteria (baseline heart rate $\geq 75 \mathrm{bpm}$ $(\mathrm{n}=4154))$ in order to avoid breaking randomisation and reducing
Table 1 Key model assumptions

\begin{tabular}{ll}
\hline Parameter description & Base case value \\
\hline Model structure & Two state Markov \\
& cohort model \\
Modelled cycle length & 1 month \\
Time horizon & Lifetime \\
Costs and effects discount rate per annum & $3.50 \%$ \\
Parametric survival model CV mortality & Gompertz \\
Extrapolation CV mortality post trial & Gompertz \\
Regression model hospitalisation & Poisson \\
Regression model NYHA class & Generalised ordered \\
& logistic \\
Regression model QoL & Multilevel model \\
Drug costs per month (f) & \\
Standard care average cost per month & 9.54 \\
Ivabradine average cost per month & 42.10 \\
Other therapy related costs ( $\mathrm{f}$ ) & \\
ECG unit cost & 31.28 (12.01-44.30) \\
CV specialist visit unit cost & 118.81 (89.48-138.97) \\
Hospitalisations cost per event ( $\mathrm{f}$ ) & \\
HF diagnosis (general ward) & 2307.98 \\
HF diagnosis (cardiac ward) & 3295.12 \\
Other CV diagnosis (general ward) & 1942.44 \\
Other CV diagnosis (cardiac ward) & 1729.60 \\
Non-CV diagnosis (general ward) & 2643.56 \\
Admission type given hospitalisation & \\
Proportion of hospitalised patients admitted in & $50 \%$ (40-60\%) \\
cardiac specialist ward versus general ward & \\
Other resource use & 26.77 \\
Ongoing HF management costs per month & \\
\hline Regression equations reported in online supplementary technical appendix. \\
Ivabradine average dose=6.7794 mg, British National Formulary list price $\mathrm{f} 40.17$ per \\
pack. \\
HF hospitalisation weighted average of HRG (Health Resource Group) codes: EB03H- \\
EBO3I. \\
Cardiovascular hospitalisation weighted average HRG codes: EA03Z-EB10Z. \\
All-cause hospitalisation HRG weighted average codes AA02Z-WA23Y. \\
CV, cardiovascular; HF, heart failure; NYHA, New York Heart Association; QoL, \\
quality of life. & \\
&
\end{tabular}

the predictive power of the risk equations due to the smaller sample size.

The risk of non-CV mortality has been estimated using age and sex adjusted UK national life table data with $\mathrm{CV}$ mortality removed. ${ }^{10}$

\section{NYHA class}

The most commonly used classification of HF severity is the NYHA classification of functional capacity which assigns patients to one of four classes depending on patient symptoms. ${ }^{11}$ In each monthly cycle, the patients who remained alive were distributed into one of the four NYHA classes using a risk equation developed from $\mathrm{SHI}_{\mathrm{f}} \mathrm{T}$ individual patient data. NYHA class has been captured in the cost effectiveness analysis primarily to determine the potential quality of life of patients over time, since NYHA class was found to strongly predict patients' quality of life. In the post-trial period the proportion of patients in each NYHA class is assumed to remain fixed (although in absolute terms numbers in each category vary according to survival estimates). This approach was taken because an extrapolation based on $\mathrm{SHI}_{\mathrm{f}} \mathrm{T}$ data predicted that the proportion of patients with minimal or mild symptoms would increase over time, consistent with trends in $\mathrm{SHI}_{\mathrm{f}} \mathrm{T}$ observed data, which for the long-term were not considered clinically plausible. 


\section{Patients' quality of life}

The $\mathrm{SHI}_{\mathrm{f}} \mathrm{T}$ patient reported outcomes substudy collected quality of life (QoL) data using the EuroQoL (EQ-5D) questionnaire, which was administered to patients in countries with a validated questionnaire $(n=5313)$. The EQ-5D is a generic instrument designed to capture patient reported outcomes across five health domains (self-care, mobility, usual activities, pain/discomfort, anxiety/depression). ${ }^{12}$ QoL weights (utility values) may be derived from the EQ-5D using country-specific values of different health statuses; UK values were used in this study regardless of the country of origin of the QoL data. Utility values typically measure patient QoL on a scale, where 0 represents death and 1 represents full health, although negative values are feasible. ${ }^{13}$ Measurements from the same individual are much more likely to be correlated than estimates from different individuals and it is important to take into account such correlation when analysing data with repeated measures to increase precision and avoid bias. EQ-5D data have been analysed using multilevel modelling, a regression technique appropriate for repeated observations across individuals. The regression equation was designed to predict patient utility according to treatment allocation, baseline characteristics, NYHA class (time varying) and a hospitalisation episode.

\section{Resource use and costs}

The cost per month for ivabradine therapy ( $£ 42.10$ per month) was estimated using British National Formulary (BNF) list prices $(5 \mathrm{mg} / 7.5 \mathrm{mg}=£ 0.72$ per tablet, $2.5 \mathrm{mg}$ (half a $5 \mathrm{mg}$ tablet= £0.36)) multiplied by the distribution of patients taking each dose in $\mathrm{SHI}_{\mathrm{f}} \mathrm{T}$ (approximately $7 \% 2.5 \mathrm{mg}$, and 93\% $5 \mathrm{mg}$ or $7 \mathrm{mg}$ ). An additional one-off titration visit and ECG has been included as an administration cost for ivabradine patients. It was assumed that, once titrated, patients would be monitored at routine clinical assessments, hence further ongoing administration costs were not included for ivabradine. ${ }^{14}$ Standard therapy use ( $£ 9.54$ per month) was also estimated, using BNF list prices and the proportion of patients treated with each therapy in $\mathrm{SHI}_{\mathrm{f}} \mathrm{T}$ ( $\beta$-blockers (89\%), ACE inhibitors and/or angiotensin receptor blockers (ARBs) (91\%), aldosterone antagonists (60\%) and diuretics (83\%)). The analysis includes costs for hospitalisations by admission type (HF, other $\mathrm{CV}$ and non-CV) estimated from UK NHS data. ${ }^{14}$ Unit costs were reported for a 2011 cost year, inflation-adjusted where necessary using the health component of the UK consumer price index. ${ }^{15}$ Resource use quantities and unit costs are summarised in table 1.

\section{Sensitivity analysis}

A series of one-way sensitivity analyses on parameter values and structural assumptions have been undertaken to test the robustness of model results to changes in individual model parameters while remaining assumptions were held constant. NICE recommends that a range of sensitivity analyses should be undertaken to reflect alternative assumptions for the treatment effect for the intervention of interest, particularly regarding the modelled benefit in the post-trial period. In our analyses we have explored the ivabradine treatment effect using a range of sensitivity analyses. These include variation of ivabradine's treatment effect within 95\% CIs (CV mortality, hospitalisation and QoL), variation in the duration of the effect (gradual reduction until no further benefit of therapy is assumed over a 5-year and a 10 -year range while assuming lifelong costs) and restricting the ivabradine treatment effect to $\mathrm{HF}$ mortality and $\mathrm{HF}$ hospitalisation end points only (rather than to the broader CV mortality and all-cause hospitalisation end point).

Other sensitivity analyses for mortality included the use of alternative distributions (exponential and Weibull) for the parametric survival analysis and the use of alternative external data to predict mortality in the post-trial period. ${ }^{16}{ }^{17}$ Sensitivity analyses conducted on the hospitalisation end point included doubling and halving the rate of hospitalisation and applying alternative UK data for hospitalisation length of stay (National HF Audit and Hospital Episode Statistics data ${ }^{18}{ }^{19}$ ). Quality of life estimates were explored using alternative utility estimates from external sources. ${ }^{20}$ The distribution of patients in each NYHA class in the post-trial period was explored using a modelled scenario in which patients' symptoms were assumed to deteriorate over time $(5 \%$ of patients were redistributed each year into NYHA classes associated with more severe HF symptoms).

Probabilistic sensitivity analysis has been used to assess overall parameter uncertainty in the model. In this analysis point estimates for each parameter have been replaced with values sampled from statistical distributions and the ICER has been recalculated using the new resampled values. ${ }^{21}$ This process has been repeated 1000 times to predict the likelihood that ivabradine would be cost-effective at different cost effectiveness thresholds (the value the decision maker is willing to pay for each additional QALY).

\section{Subgroup analyses}

Subgroup analyses have been performed for subgroup populations identified from the clinical study protocol. These included age ( $<$ or $\geq 75$ years old); HF duration (categoried by quartile cut points); NYHA class; LVEF (categoried by quartile cut points); prior ischaemia; prior diabetes and $\beta$-blocker use.

\section{RESULTS}

\section{Base case analysis}

A parametric model based on a Gompertz distribution was established as the best fit of the observed data based on statistical evidence (Akaike and Bayesian information), a visual review of Kaplan-Meier survival plots versus predicted curves and the plausibility of predicted survival in the extrapolated, post-trial period (see online supplementary technical appendix). The parametric survival analysis predicted that mean survival for ivabradine patients with a heart rate $\geq 75 \mathrm{bpm}$ would be 5.61 years compared with 5.86 years for standard care patients. Ivabradine was expected to improve patient survival duration by 0.25 years (approximately 3.0 months) compared with standard care. Our analysis suggests that, over 1 year, approximately 225 patients would need to be treated to prevent one HF death.

Quality of life scores for the base case analysis ranged from 0.82 to 0.46 for standard care patients and from 0.84 to 0.47 for ivabradine patients (based on NYHA classes I-IV, and no hospitalisation event). QoL increased, on average, by 0.014 due to ivabradine therapy itself. However, hospitalisations were associated with a substantial temporary QoL loss, which varied according to NYHA class ( -0.04 to -0.29 ; NYHA I-IV). Ivabradine therapy was also associated with a reduction in hospitalisations and hence also avoided the potential QoL loss associated with these events, see table 2. Overall, in a lifetime analysis, ivabradine plus standard care was associated with a gain of 0.28 QALYs (approximately 3.4 quality adjusted life months) versus standard care alone.

The model predicted that, over a lifetime, ivabradine would be $£ 2376$ more expensive per patient compared with standard 
Table 2 Utility values predicted for $\mathrm{SHI}_{\mathrm{f}} \top$ population heart rate $\geq 75 \mathrm{bpm}$

\begin{tabular}{lc}
\hline Description & Utility value \\
\hline No hospitalisation & 0.82 \\
NYHA I & 0.74 \\
NYHA II & 0.64 \\
NYHA III & 0.46 \\
NYHA IV & \\
Hospitalisation & -0.04 \\
NYHA I & -0.07 \\
NYHA II & -0.10 \\
NYHA III & -0.29 \\
NYHA IV & 0.01 \\
Ivabradine & \\
\hline Regression equation for quality of life estimates reported in online supplementary \\
technical appendix. & \\
Utility values estimated using EQ-5D data; UK tariff values. & \\
NYHA, New York Heart Association.
\end{tabular}

care alone. The additional drug therapy and follow-up costs ( $£ 3341$ per patient) were offset by an important reduction in expected hospitalisation costs ( $£ 965$ per patient). In HF, with a heart rate $\geq 75 \mathrm{bpm}$, ivabradine would be expected to reduce the rate of HF hospitalisation from approximately 18 hospitalisations per 1000 patient months (standard care) to 13 hospitalisations per 1000 patient months (ivabradine plus standard care); 20 patients would need to be treated to prevent one HF hospitalisation.

The incremental cost per additional QALY for ivabradine plus standard care versus standard care has been estimated as $£ 8498$ for heart rate $\geq 75 \mathrm{bpm}$ and $£ 13764$ for heart rate $\geq 70 \mathrm{bpm}$.

\section{Subgroup analyses}

The cost per QALY increased by $20 \%$ in patients on target dose $\beta$-blocker therapy due to their lower risk of mortality and hospitalisation and a slightly lower heart rate compared with patients not on target dose therapy. Despite this increase, ivabradine remains cost-effective at existing NICE cost effectiveness threshold values. The ICER for patients on target dose $\beta$-blocker therapy is estimated as $£ 10374$ per QALY (heart rate $\geq 75 \mathrm{bpm}$ ) and $£ 16578$ per QALY (heart rate $\geq 70 \mathrm{bpm}$ ).

\section{Deterministic sensitivity analysis}

The Tornado diagram in figure 1 shows the effect on the estimated ICER if one model assumption is altered while other assumptions/parameter values remain at base case values. The ICER remains below $£ 20000$ in virtually all scenarios. The ICER was sensitive to changes in the treatment effects of ivabradine at the upper bound 95\% CI for CV mortality (HR 0.80 to 1.03). However, the risk equations were developed using data from the total $\mathrm{SHI}_{\mathrm{f}} \mathrm{T}$ population (heart rate $\geq 70 \mathrm{bpm}$ ), therefore, this scenario analysis overestimates the upper bound HR and ICER estimate for the licensed indication (heart rate $\geq 75 \mathrm{bpm}$ ). A scenario analysis which modelled costs and outcomes only for the $\mathrm{SHI}_{\mathrm{f}} \mathrm{T}$ trial follow-up period resulted in an ICER of $£ 15175$. This estimate was less favourable than the base case estimate because the short time horizon did not take into account long-term benefits associated with ivabradine.

\section{Probabilistic sensitivity analysis}

Probabilistic sensitivity analysis indicates that, using a cost effectiveness threshold of $£ 20000$ per QALY, ivabradine plus standard care has a greater than 0.95 probability of being costeffective versus standard care alone in a population with a heart rate $\geq 75 \mathrm{bpm}$ (figure 2 ), and over 0.70 probability in a population $\geq 70 \mathrm{bpm}$.

\section{DISCUSSION}

The cost effectiveness analysis suggests that ivabradine plus standard care has a high probability of being cost-effective versus standard care alone in patients with HF who are in sinus rhythm with left ventricular systolic dysfunction and have a baseline heart rate either $\geq 75 \mathrm{bpm}$ or $\geq 70 \mathrm{bpm}$. The cost effectiveness of ivabradine is driven by important reductions in HF mortality and hospitalisation and associated costs of care as well as improvements in QoL.

Probabilistic sensitivity analyses suggest that, at the current lower bound NICE cost effectiveness threshold range, ivabradine plus standard care therapy has a 0.95 probability of being costeffective versus standard care alone in a population consistent with the European licensed indication (heart rate $\geq 75 \mathrm{bpm}$ ), and over a 0.70 probability in the entire $\mathrm{SHI}_{\mathrm{f}} \mathrm{T}$ study population (heart rate $\geq 70 \mathrm{bpm}$ ). The cost effectiveness results were robust to a range of sensitivity analyses that tested alternative assumptions for parameter values and model structure. Our analyses also indicated that ivabradine would be expected to remain effective in a range of patient subgroups including those on target dose $\beta$-blockade. A separate budget impact analysis undertaken by NICE indicated that the total budget impact of ivabradine in the UK would be expected to be approximately $£ 4400$ per 100000 people. $^{22}$

The cost effectiveness analysis has been developed using individual patient data from a pivotal, large scale randomised controlled trial $\mathrm{SHI}_{\mathrm{f}} \mathrm{T}$. Model results have been shown to be robust and calibrate well against observed patient data.

It is acknowledged that that the cost effectiveness result for patients on target dose $\beta$-blockade may appear to contradict simple univariable analyses based on $\mathrm{SHI}_{\mathrm{f}} \mathrm{T}$ data that indicated that ivabradine was not associated with a statistically significant treatment effect on either mortality or the rate of hospitalisation in these patients. $\mathrm{SHI}_{\mathrm{f}} \mathrm{T}$ patients on target dose $\beta$-blockade were found to be generally healthier and at a low risk of mortality and hospitalisation. Furthermore, there were only $15 \mathrm{HF}$ deaths (standard care) and $10 \mathrm{HF}$ deaths with ivabradine plus standard care in patients on target dose therapy with heart rate $\geq 75 \mathrm{bpm}$ $(n=938)$. While the HR of patients on target dose therapy (HR: 0.68 ) was close to the HR observed in the base case population (HR: 0.62), the underlying clinical event rate was insufficient to provide statistical significance. It is also noted that the baseline characteristics of patients on target dose $\beta$-blockade in the ivabradine treatment group differed from those patients on target dose therapy in the standard care treatment group. In isolation, univariable analyses may consequently provide a misleading picture of the ivabradine treatment effect given a low underlying clinical event rate, small sample size in this subgroup and no correction for clinical differences at baseline between patients. A strength of this study is that it is based on multivariable risk equations which take into account the change in efficacy of ivabradine by baseline heart rate and adjust for differences in key prognostic risk factors. Our analyses indicated that there was no evidence that the treatment effect of ivabradine was altered by $\beta$-blocker use, ischaemia or age once differences in baseline heart rate had been taken into account $(p>0.05)$. This suggested that, if patient heart rate remained high despite target $\beta$-blocker dose, ivabradine has a beneficial effect. Although the underlying clinical event rate in patients on target dose $\beta$-blocker therapy may be low, ivabradine is nevertheless 


\section{Tornado Diagram: Sensitivity Analyses Parameter Estimates}

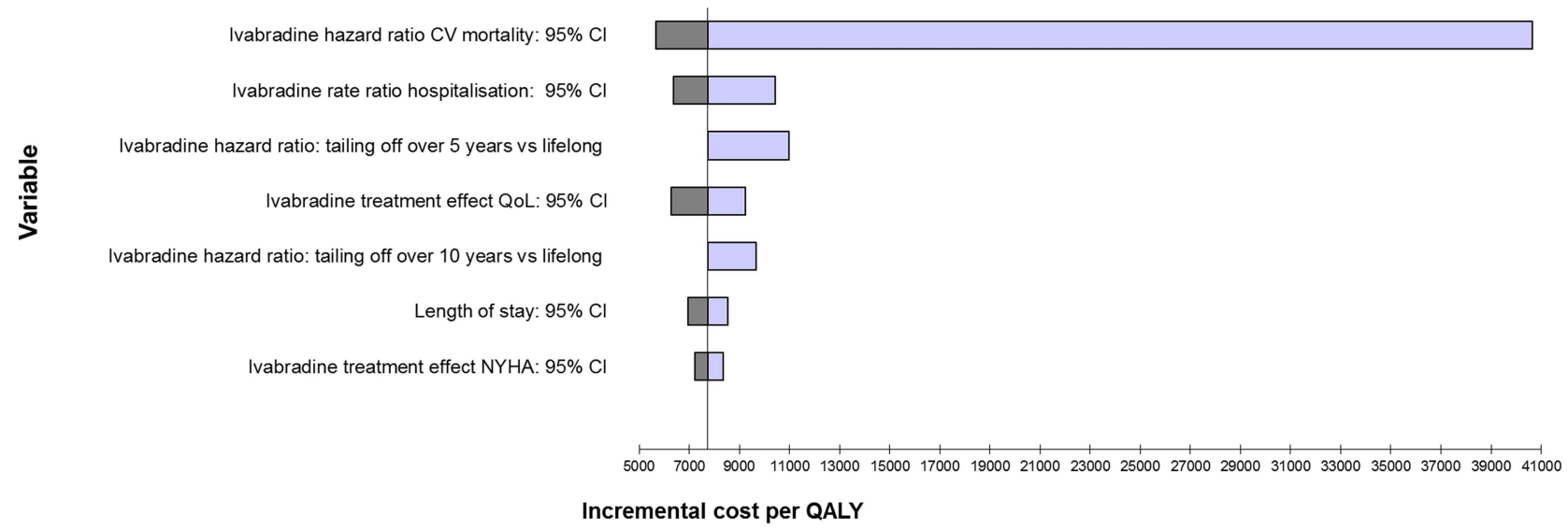

Tornado Diagram: Sensivity Analyses Structural Assumptions

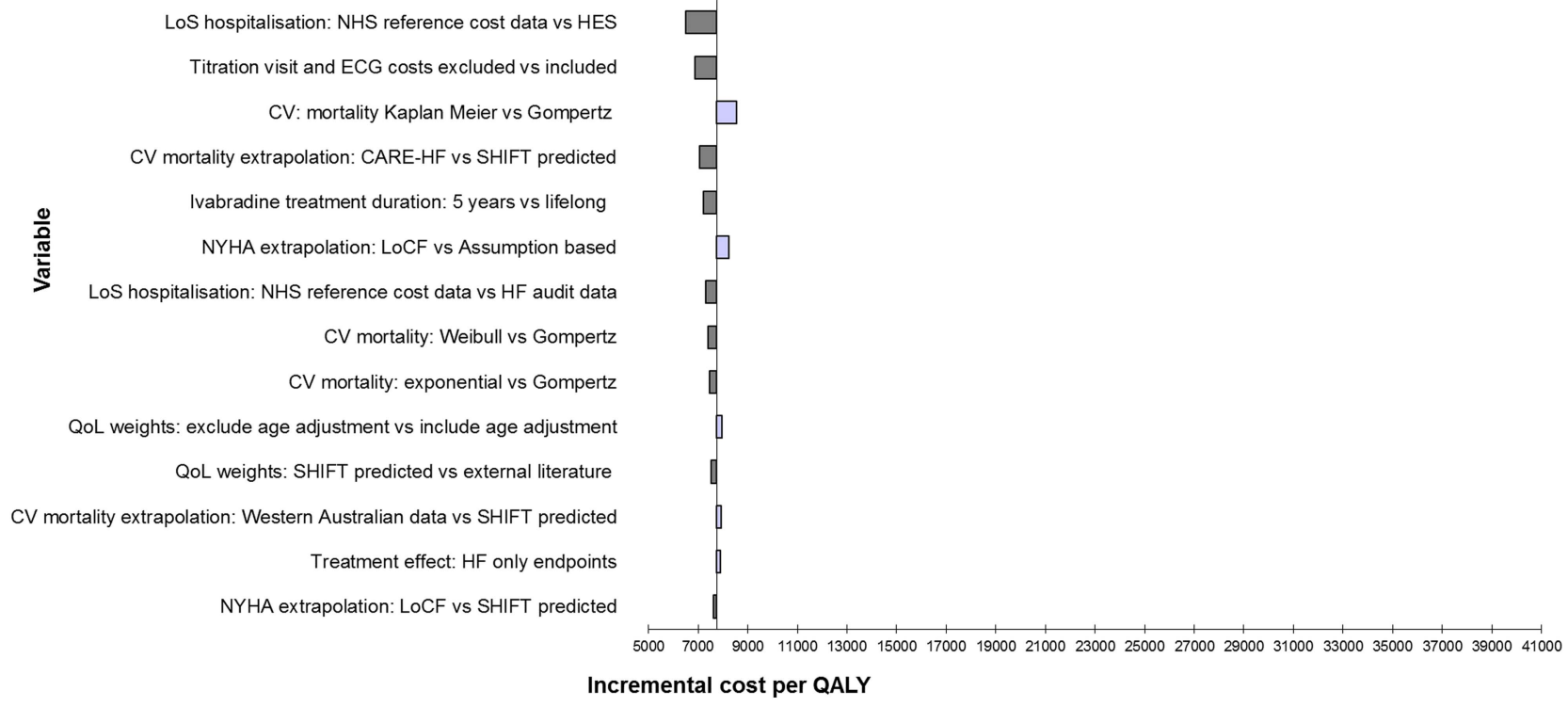

Figure 1 One way sensitivity analyses for patients with heart failure (HF), heart rate $\geq 75 \mathrm{bpm}$ ( $\mathrm{f}$ ).

expected to reduce mortality and hospitalisations in such patients relative to standard care.

These analyses were designed to derive the most likely estimate of effect for ivabradine in specific patient subgroups including those patients on target dose $\beta$-blocker therapy based on available evidence from $\mathrm{SHI}_{\mathrm{f}} \mathrm{T}$. An alternative approach to determining the clinical effect of ivabradine on top of target dose $\beta$-blockade would be to conduct a randomised trial in this population. However, such a trial would have to be large, as those patients who tolerate target dose $\beta$-blockade in $\mathrm{SHI}_{\mathrm{f}} \mathrm{T}$ appeared to be the healthiest, lowest-risk patients hence the underlying event rate in these patients is likely to be low.

In summary, from a UK NHS perspective, ivabradine in combination with optimised standard care therapy, including $\beta$-blockade,

Table 3 Base-case results in patients with heart rate $\geq 75 \mathrm{bpm}$ and $\geq 70 \mathrm{bpm}$

\begin{tabular}{|c|c|c|c|c|c|c|c|c|c|}
\hline Population & Technologies & $\begin{array}{l}\text { Total } \\
\text { costs (f) }\end{array}$ & $\begin{array}{l}\text { Total } \\
\text { LYs }\end{array}$ & $\begin{array}{l}\text { Total } \\
\text { QALYs }\end{array}$ & $\begin{array}{l}\text { Incremental } \\
\text { costs (f) }\end{array}$ & $\begin{array}{l}\text { Incremental } \\
\text { LYs }\end{array}$ & $\begin{array}{l}\text { Incremental } \\
\text { QALYs }\end{array}$ & $\begin{array}{l}\text { ICER (f) } \\
\text { incremental LYs }\end{array}$ & $\begin{array}{l}\text { ICER (f) } \\
\text { incremental } \\
\text { QALY }\end{array}$ \\
\hline Heart rate & Standard care & 9446 & 5.61 & 3.99 & - & - & - & - & - \\
\hline$\geq 75$ bpm & $\begin{array}{l}\text { Ivabradine plus std } \\
\text { care }\end{array}$ & 11822 & 5.86 & 4.27 & 2376 & 0.25 & 0.28 & 9363 & 8498 \\
\hline Heart rate & Standard care & 9312 & 5.89 & 4.23 & & - & - & - & - \\
\hline$\geq 70 \mathrm{bpm}$ & $\begin{array}{l}\text { Ivabradine plus } \\
\text { standard care }\end{array}$ & 11796 & 6.03 & 4.41 & 2484 & 0.14 & 0.18 & 17875 & 13764 \\
\hline
\end{tabular}




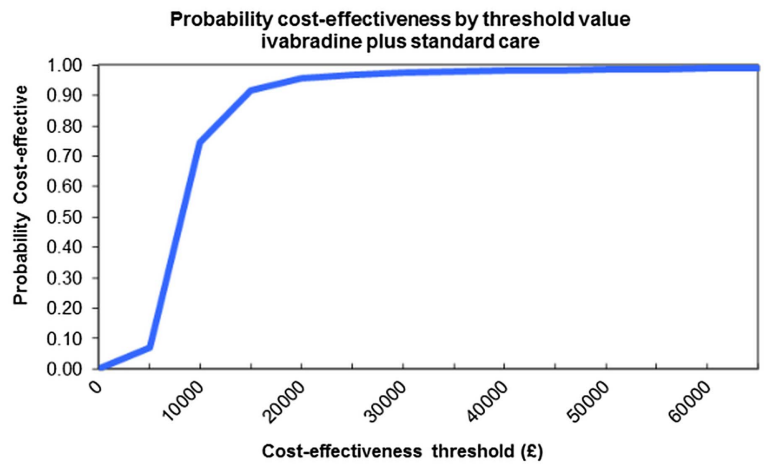

Figure 2 The cost effectiveness acceptability curve for patients with heart failure (HF), heart rate $\geq 75 \mathrm{bpm} \mathrm{(f).}$

has a high probability of being cost-effective versus standard care alone in patients with HF, who are in sinus rhythm with left ventricular systolic dysfunction and a baseline heart rate either $\geq 75 \mathrm{bpm}$ or $\geq 70 \mathrm{bpm}$. In support of this, the single technology appraisal of ivabradine by NICE concluded that this analysis was robust and unlikely to overestimate the cost effectiveness, leading to a recommendation that patients in England and Wales should have access to this therapy if indicated. ${ }^{9}$

\section{Key messages}

\section{What is already known about this subject?} Ivabradine, a specific heart rate lowering therapy, has been shown in a randomised placebo-controlled study, Systolic HF Treatment with the If Inhibitor Ivabradine Trial $\left(\mathrm{SHI}_{\mathrm{f}} \mathrm{T}\right)$, to significantly reduce the composite end point of cardiovascular death and hospitalisation for worsening heart failure (HF) in patients with systolic HF who are in sinus rhythm and with a heart rate $\geq 70 \mathrm{bpm}$, when added to optimised medical therapy.

\section{What does this study add?}

We assessed the cost effectiveness of ivabradine, from a UK National Health Service (NHS) perspective, based on the results of $\mathrm{SHI}_{\mathrm{f}} \mathrm{T}$. The incremental cost per additional quality adjusted life year (QALY) for ivabradine plus standard care versus standard care has been estimated as $\mathrm{f} 8498$ for heart rate $\geq 75 \mathrm{bpm}$ and $\mathrm{f13} 764$ for heart rate $\geq 70 \mathrm{bpm}$. Ivabradine is expected to have a $95 \%$ chance of being cost-effective in the EU licensed population using the current National Institute of Health and Care Excellence (NICE) cost effectiveness threshold of £20 000 per QALY.

\section{How might this impact on clinical practice?}

These results should encourage clinicians to prescribe ivabradine to appropriate patients, as it has a clinical effect that represents good value for money when added to optimised medical therapy.

Acknowledgements MRC's salary is supported by the UK National Institute for Health Research Cardiovascular Biomedical Research Unit at the Royal Brompton Hospital, London.

Contributors All authors contributed to the planning, conduct and reporting of the work described in this article. MRC and MS are guarantors of the overall content.
Funding This work was supported by The Servier Research Group.

Competing interests MRC reports receiving consultancy and speaking fees from Servier. ICON Health Economics was contracted to undertake the analysis and has supported AG, NP, AD and MS through salary or consultancy payments. NB is a Servier employee.

Ethics approval $\mathrm{SH}_{\mathrm{f}} \mathrm{T}$ trial provided the data for this analysis: approval from all countries' ethical approval bodies as per GCP.

Provenance and peer review Not commissioned; externally peer reviewed.

Data sharing statement Further data are available in the online supplementary appendix. Requests for additional data should be sent to Servier laboratories for discussion with the Executive Steering Committee of the $\mathrm{SH}_{\mathrm{f}} \mathrm{T}$ Study.

Open Access This is an Open Access article distributed in accordance with the Creative Commons Attribution Non Commercial (CC BY-NC 3.0) license, which permits others to distribute, remix, adapt, build upon this work non-commercially, and license their derivative works on different terms, provided the original work is properly cited and the use is non-commercial. See: http://creativecommons.org/ licenses/by-nc/3.0/

\section{REFERENCES}

1 British Heart Foundation. Coronary heart disease statistics. BHF, 2012. http://www. heartstats.org

2 British Heart Foundation. Heart health conditions: heart failure. BHF, 2013. http:/l www.bhf.org.uk

3 NICE. Chronic Heart Failure- National clinical guideline for diagnosis and management in primary and secondary care. 2010.

4 Kjekshus J, Gullestad L. Heart rate as a therapeutic target in heart failure. Eur Heart J 1999;1:H64-9.

5 Black J. Drugs from emasculated hormones: the priniciples of syntopic antagonism. Bio Rep 1989;9:253-72.

6 Swedberg K, Komajda M, Böhm M, et al. Effects on outcomes of heart rate reduction by ivabradine in patients with congestive heart failure: is there an influence of beta-blocker dose?: findings from the SHIfT study. J Am Coll Cardiol 2012;59:1938-45.

7 Böhm M, Borer J, Ford I, et al. Heart rate at baseline influences the effect of ivabradine on cardiovascular outcomes in chronic heart failure. Analysis from the $\mathrm{SHI}_{\mathrm{f}} \mathrm{T}$ study. Clin Res Cardiol 2012;10:1-12.

8 Komajda M, Follath F, Swedberg K, et al. The EuroHeart Failure Survey programme-a survey on the quality of care among patients with heart failure in Europe. Eur Soc Cardiol 2003;24:464-74.

9 National Institute of Health and Care Excellence (NICE). Guide to the methods of technology appraisal. London: National Institute of Health Care Excellence, 2013.

10 Office of National Statistics. Period expectation of life. Office of National Statistics, 2010. http://www.statistics.gov.uk

11 New York Heart Association. New York Heart Association (NYHA) functional classification: the criteria committee of the New York heart association. Nomenclature and criteria for diagnosis of diseases of the heart and great vessels. Boston, Mass: Little, Brown \& Co, 1994. Report No.: 9th ed.

12 Euroqol group. EQ-5D. EuroQol Group, 2011. http://www.euroqol.org/eq-5d/ reference-search/reference-search.html

13 Twisk J. Applied longitudinal data analysis for epidemiology: a practical guide. Cambridge University Press, 2003.

14 The Department of Health. NHS schedule of reference costs 2010/2011. The Department of Health, 2011. http://www.dh.gov.uk

15 Office of National Statistics. Consumer price index. Office of National Statistics, 2011. http://www.statistics.gov.uk/hub/index.html

16 Cleland J. CARE-HF long term follow up [abstract]. Eur Soc Cardiol 2011.

17 Teng K, Hung J, Knuiman $\mathrm{M}$, et al. Trends in long-term cardiovascular mortality and morbidity in men and women with heart failure of ischaemic versus non-ischaemic aetiology in Western Australia betweeen 1990 and 2005. Int J Cardiol 2011;158:405-10.

18 The Information Centre for health and social care. Hospital episode statistics online. National Health Service, 2012. http://www.hesonline.nhs.co.uk

19 National Institute for Cardiovascular Outcomes Research (NICOR). National Heart Failure Audit. 2011.

20 Golher A, Geisler B, Manne J, et al. Utility estimates for decision-analytic modeling in chronic heart failure-health states based on the NYHA classes and number of rehospitalisations. Value Health 2009;12:185-7.

21 Briggs A, Claxton K, Sculpher M. Decision modelling for health economic evaluation. Oxford: Oxford University Press, 2006.

22 National Institute of Health and Care Excellence. Ivabradine for treating chronic heart failure: costing template. NICE technology appraisal 267, 2012. http:/l guidance.nice.org.uk/TA267/CostingTemplate/xIs/English 